\title{
Ytterbium Intercalation of Epitaxial Graphene Grown on Si-Face SiC
}

\author{
Somsakul Watcharinyanon ${ }^{1}$, Leif I. Johansson ${ }^{1}$, Chao Xia ${ }^{1}$, Jan Ingo Flege ${ }^{2}$, \\ Axel Meyer ${ }^{2}$, Jens Falta ${ }^{2}$, Chariya Virojanadara ${ }^{1}$ \\ ${ }^{1}$ Department of Physics, Chemistry, and Biology, Linköping University, Linköping, Sweden \\ ${ }^{2}$ Institute of Solid State Physics, University of Bremen, Bremen, Germany \\ Email: somwat@ifm.liu.se
}

Received February 12, 2013; revised March 14, 2013; accepted April 9, 2013

Copyright (C) 2013 Somsakul Watcharinyanon et al. This is an open access article distributed under the Creative Commons Attribution License, which permits unrestricted use, distribution, and reproduction in any medium, provided the original work is properly cited.

\begin{abstract}
The rare-earth metal, ytterbium (Yb), was deposited on graphene grown on Si-face SiC, kept at room temperature. $\mathrm{Yb}$ was not found to intercalate, destroy or dope the graphene layer before subsequent heating, unlike alkali metals such as $\mathrm{Li}$ and $\mathrm{Na}$. Our photoemission results reveal that heating to $300^{\circ} \mathrm{C}$ promotes $\mathrm{Yb}$ intercalation into the graphene-substrate interface. Real-time low energy electron microscopy (LEEM) measurements indicated intercalation to start at a sample temperature of around $220^{\circ} \mathrm{C}$. In the intercalation process, $\mathrm{Yb}$ penetrates through the graphene and buffer layer and forms bonds to the silicon in the topmost $\mathrm{SiC}$ substrate bilayer, as indicated by the shifted components observed in the $\mathrm{C} 1 \mathrm{~s}$, Si 2p, and $\mathrm{Yb} 4 \mathrm{f}$ spectra. The $\mathrm{Yb}$ intercalation decouples the buffer layer from the substrate and transforms it into another graphene layer as manifested by the absence of buffer layer spots in the $\mu$-LEED pattern and the appearance of an additional $\pi$ band in the ARPES spectra, respectively. Moreover, the observed shift of the Dirac point down from the Fermi level by $1.9 \mathrm{eV}$ indicates electron doping of the graphene layer upon $\mathrm{Yb}$ intercalation. The Yb intercalated graphene sample was found to be thermodynamically stable up to temperatures around $700^{\circ} \mathrm{C}$.
\end{abstract}

Keywords: Epitaxial Graphene; SiC; Ytterbium; Intercalation; PES; ARPES; LEEM

\section{Introduction}

The intercalation of graphene has attracted considerable recent interest, and has been studied quite intensively, mainly due to ongoing developments of graphene-based electronics. Intercalation has shown to provide the capability to modify the structural and electronic properties of graphene, i.e. to make it possible to tailor the properties of graphene to meet the need of specific applications. A monolayer/zero-layer graphene grown on $\mathrm{SiC}(0001)$ substrate can be transformed into bi-layer/monolayer graphene upon hydrogen $(\mathrm{H})$ intercalation [1-3]. H can go underneath the graphene and the buffer layer [4], which is a carbon layer located in between graphene layers and $\mathrm{SiC}(0001)$ substrate with graphene-like honeycomb structure but no graphitic electronic properties. This decouples the buffer layer from the substrate and transforms it into another graphene layer. $\mathrm{H}$ intercalation is found to induce $p$-type doping of the graphene layer, shifting the Fermi level below the Dirac point, i.e. the crossing point of the $\pi$ cone(s) at the $\bar{K}$ point(s) in the graphene hexagonal Brillouin zone. Furthermore the $\mathrm{H}$ intercalation has been reported to enhance the charge carrier mobility of graphene on $\mathrm{SiC}(0001)$ [5]. Some of the alkali metals such as lithium (Li) [6,7] and sodium (Na) $[8,9]$ have also been shown to intercalate graphene on $\mathrm{SiC}(0001)$ and decouple the carbon buffer layer from the $\mathrm{SiC}$ substrate. Alkali metal intercalation provides strong $n$-doping of the graphene, so the Dirac point then becomes located well below the Fermi level. Apart from these elements, noble metals like gold $(\mathrm{Au})$ [10], group IV and VII elements like silicon ( $\mathrm{Si}$ ) [11], germanium (Ge) [12] and fluorine (F) [13] have been used for intercalation of graphene on $\mathrm{SiC}(0001)$ and were shown to have a large influence on the structural and electronic properties. Intercalation of graphene grown on transition metal substrates has also been investigated [14,15]. In this case the intercalation gives rise to a weakening of the interaction between graphene and the metal substrate. Although a large variety of elements have been shown to successfully intercalate into graphene, earlier studies have shown that some alkali metals such as rubidium $(\mathrm{Rb})$ and cesium (Cs) [16] do not intercalate graphene on $\mathrm{SiC}(0001)$ and do not decouple the buffer layer and, apparently, neither does potassium $(\mathrm{K})[17,18]$. 
We have introduced a rare-earth metal, ytterbium (Yb), to perform the intercalation of graphene grown on $\mathrm{SiC}$ (0001). From a technical point of view, $\mathrm{Yb}$ is well known for its magnetic properties and potential for magnetic storage applications [19]. The possibility to use $\mathrm{Yb}$ for intercalation of graphite has earlier been studied and reported [20,21]. A. M. Shinkin et al. demonstrated that the $\mathrm{Yb}$ deposition onto single-crystalline graphite flakes [20] or monolayer graphite grown on $\mathrm{Ni}(111)$ [21] followed by subsequent heating results in the intercalation of $\mathrm{Yb}$. They established that a heating temperature of $250^{\circ} \mathrm{C}$ $400^{\circ} \mathrm{C}$ was the most efficient range for the $\mathrm{Yb}$ intercalation process. The $\mathrm{Yb}$ graphite intercalation compound has been reported to exhibit superconducting behavior [22]. Photoemission data collected from a single crystalline $\mathrm{Yb}$ graphite intercalation compound indicated charge transfer from the $\mathrm{Yb}$ atoms into vacant graphitederived $\pi$ states, resulting in a lowering of the $\pi$ bands around the $\bar{K}$ point of the Brillouin zone [20]. A lowering of the $\pi$ bands was also observed for $\mathrm{Yb}$ inter calated graphite on $\mathrm{Ni}(111)$, but it was described [21] to arise from hybridization of the graphite $\pi$ states with $d$ states of the metal substrate. In the present work, we study the possibility of $\mathrm{Yb}$ intercalation of graphene grown on $\mathrm{SiC}(0001)$ by using photoemission and angleresolved photoelectron spectroscopy (PES and AR-PES), low energy electron microscopy (LEEM) and selected area low energy electron diffraction obtained by restricted illumination ( $\mu$-LEED). The results show that no intercalation occurs after deposition of $\mathrm{Yb}$ on the sample at room temperature. Subsequent heating is shown to be required to promote $\mathrm{Yb}$ intercalation similar to what was found earlier for graphite and graphene on $\mathrm{Ni}(111)$ [20, 21]. We find that a heating temperature of about $220^{\circ} \mathrm{C}$ is required for the $\mathrm{Yb}$ intercalation process to occur for graphene on $\mathrm{SiC}(0001)$. Our experimental results reveal that $\mathrm{Yb}$ intercalation induces significant changes in the electronic properties of the graphene layer(s).

\section{Experimental}

Graphene was prepared by direct current heating of $n$ type on-axis $4 \mathrm{H}-\mathrm{SiC}(0001)$ substrates at a temperature of $1300^{\circ} \mathrm{C}$ for two minutes in ultra-high vacuum. This method is known to provide a dominant coverage of 1 monolayer (ML) graphene with a mixture of small 0 and 2 ML areas/domains [23-25], which was also verified by PES. Deposition of $\mathrm{Yb}$ on the graphene samples was performed in situ by resistive heating of a tungsten coil. The substrates were kept at room temperature during deposition. The base pressure of the system was in the low $10^{-10}$ mbar range. The electronic structure was then investigated using PES and ARPES while the sample was heated step by step from $300^{\circ} \mathrm{C}$ to $900^{\circ} \mathrm{C}$. Similarly prepared samples were also investigated using LEEM and $\mu$-LEED, which allowed probing of the morphology and structure of the samples in real-time during the heating process.

The XPS and ARPES experiments were carried out at the synchrotron radiation facility MAX-lab in Lund (Sweden) using beam lines $\mathrm{I} 311$ and I4, respectively. Beamline I311 is equipped with a modified SX700 monochromator and Scienta electron analyzer. It was utilized primarily for high-resolution studies of the $\mathrm{C} 1 \mathrm{~s}, \mathrm{Si}$ $2 \mathrm{p}$, and $\mathrm{Yb} 4 \mathrm{f}$ core levels. A total energy resolution of $\leq 0.10 \mathrm{eV}$ for photon energies from 33 to $450 \mathrm{eV}$ and $\leq 0.30 \mathrm{eV}$ for photon energies up to $750 \mathrm{eV}$ was selected. The ARPES experiments were performed at beamline I4. This beamline is equipped with a spherical grating monochromator and an end station with a PHIOBOS 100 two-dimensional Specs electron analyzer. LEEM and $\mu$ LEED measurements were conducted in an Elmitec LEEM III system at the University of Bremen (Germany). A spatial resolution of less than $10 \mathrm{~nm}$ can be obtained in this microscope. The base pressure of the system was about $10^{-10}$ mbar.

\section{Results and Discussion}

Upon graphene growth on $\mathrm{SiC}(0001)$ it is well known [23-25] that the graphene develops on top of a first carbon interface layer, the so-called carbon buffer layer. The C 1s core-level spectrum from a clean as-grown graphene sample (Figure 1(a), bottom curve) shows the graphene $(\mathrm{G})$ peak at a binding energy (BE) of $284.6 \mathrm{eV}$, a small shoulder at the high $\mathrm{BE}$ side originating from the carbon buffer layer $(\mathrm{B})$ and the substrate $(\mathrm{SiC})$ peak at a $\mathrm{BE}$ of $283.6 \mathrm{eV}$. The extracted $\mathrm{G} / \mathrm{SiC}$ and $\mathrm{B} / \mathrm{SiC}$ intensity ratios are found to be consistent with the values obtained earlier [26] from ex situ prepared samples with homogeneous $1 \mathrm{ML}$ graphene coverage. This suggests that the sample surface is mainly composed of $1 \mathrm{ML}$ graphene. A further confirmation of this result is provided by ARPES spectra, presented below, which show a single $\pi$ band with the Dirac point about $0.4 \mathrm{eV}$ below the Fermi level, which is characteristic of $1 \mathrm{ML}$ graphene on $\mathrm{SiC}(0001)$ [4,27]. The graphene sample was then exposed to an $\mathrm{Yb}$ flux at room temperature. After $\mathrm{Yb}$ deposition, no significant changes in the $\mathrm{C} 1 \mathrm{~s}$ spectrum were observed, see second curve from the bottom in Figure 1(a). The spectrum only appears slightly broader. Heat treatments of the sample were then performed, and a starting temperature of $300^{\circ} \mathrm{C}$ was chosen since it had been reported earlier that this temperature was the most efficient for the $\mathrm{Yb}$ intercalation process on graphite [20,21]. Distinct changes appear in the $\mathrm{C} 1 \mathrm{~s}$ spectrum directly after heating at $300^{\circ} \mathrm{C}$, see Figure 1(a). The shoulder (B) at the high $\mathrm{BE}$ side is essentially gone. Noticeable is also that the 


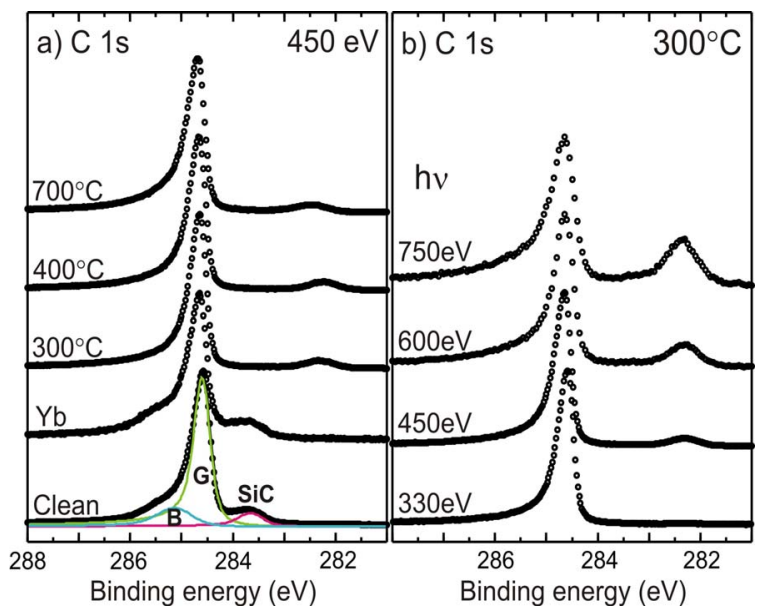

Figure 1. C 1s core level spectra collected (a) at a photon energy of $450 \mathrm{eV}$ from the monolayer graphene sample, before and after $\mathrm{Yb}$ deposition and heating from $300^{\circ} \mathrm{C}$ to $700^{\circ} \mathrm{C}$, (b) at different photon energies after $\mathrm{Yb}$ deposition and heating at $300^{\circ} \mathrm{C}$

substrate $\mathrm{SiC}$ component exhibits a shift of about $1.4 \mathrm{eV}$ towards lower BE indicating the formation of a dipole layer at the substrate graphene interface, induced by interactions between $\mathrm{Yb}$ and the $\mathrm{Si}$ atoms in the first $\mathrm{SiC}$ bilayer as also indicated in the Si $2 p$ spectrum, see below. For greater detail, the $\mathrm{C} 1 \mathrm{~s}$ spectrum was after heating at $300^{\circ} \mathrm{C}$ collected using different photon energies from 330 $\mathrm{eV}$ (surface sensitive) to $750 \mathrm{eV}$ ("bulk" sensitive) as illustrated in Figure 1(b). These spectra show a distinct increase in relative intensity of the shifted substrate $\mathrm{SiC}$ component with increasing photon energy, as expected since this component originates from the substrate underneath the graphene. After heating at $400^{\circ} \mathrm{C}$ the $\mathrm{C} 1 \mathrm{~s}$ spectrum looks very similar to that at $300^{\circ} \mathrm{C}$, see Figure 1(a). The $\mathrm{C} 1 \mathrm{~s}$ spectrum is found to be stable upon heating to $700^{\circ} \mathrm{C}$, when the shifted substrate $\mathrm{SiC}$ component starts to move back and the shoulder B on the high BE side of the graphene peak begin to reappear.

The Si $2 p$ spectrum acquired at photon energy of 190 $\mathrm{eV}$, before and after $\mathrm{Yb}$ deposition, and after heating at different temperatures is shown in Figure 2. The spectrum from the clean sample exhibits one $\mathrm{Si} 2 \mathrm{p}$ doublet $\left(2 \mathrm{p}_{1 / 2}\right.$ and $\left.2 \mathrm{p}_{3 / 2}\right)$, originating from the $\mathrm{SiC}$ substrate at a $\mathrm{BE}$ of $101.4 \mathrm{eV}\left(2 \mathrm{p}_{3 / 2}\right)$. No significant change is observed after $\mathrm{Yb}$ deposition at room temperature. Only the width of spectrum is slightly broader and the intensity has decreased somewhat. The decrease is due to the attenuation of the $\mathrm{Si} 2 \mathrm{p}$ signal by the $\mathrm{Yb}$ deposited on top of the sample. After heating at $300^{\circ} \mathrm{C}$, interestingly, the Si $2 p$ spectrum exhibits a shift to lower BE similar to what was observed for the $\mathrm{SiC}$ component in the $\mathrm{C} 1 \mathrm{~s}$ spectrum. Apart from the shift, the Si $2 \mathrm{p}$ spectrum becomes prominently broader. With a curve fitting procedure [28], applying a branching ratio of $1: 2$ and a spin-orbit splitting

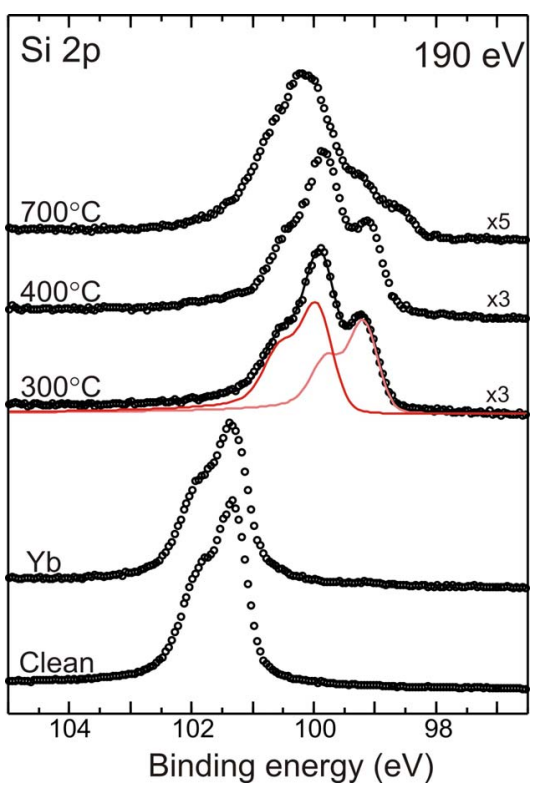

Figure 2. Si $2 p$ core level spectra collected at a photon energy of $190 \mathrm{eV}$ from the monolayer graphene sample, before and after $\mathrm{Yb}$ deposition and heating from $300^{\circ} \mathrm{C}$ to $700^{\circ} \mathrm{C}$.

of $0.61 \mathrm{eV}$, the $\mathrm{Si} 2 \mathrm{p}$ spectrum can then be decomposed into two components, the first component at a BE of 99.2 $\mathrm{eV}$ and the second component at a $\mathrm{BE}$ of $100.0 \mathrm{eV}$. The shift of the Si $2 p$ spectrum to lower BE $(99.2 \mathrm{eV})$ is interpreted to originate from an interaction between $\mathrm{Si}$ and $\mathrm{Yb}$ atoms [29] contributing to the formation of a new dipole layer at the interface. These results suggest that, at elevated temperature, $\mathrm{Yb}$ can intercalate under the graphene and the buffer layer and bond to Si atoms in thetopmost $\mathrm{SiC}$ substrate bilayer. This results in a shift to lower BE $(100.0 \mathrm{eV})$ of the SiC substrate signal. Similarly shifted Si $2 p$ components have been observed earlier from $\mathrm{H} \mathrm{[1,3],} \mathrm{Li}[6]$ and $\mathrm{Na}[8]$ intercalated graphene samples. The intercalation results in an elimination of the buffer layer as observed from the vanishing of the buffer layer (B) component in the $\mathrm{C}$ 1s spectra in Figure 1(a). After heating at $400^{\circ} \mathrm{C}$, the $\mathrm{Si} 2 \mathrm{p}$ spectrum shows similar features as observed at $300^{\circ} \mathrm{C}$. The spectrum is found to be stable up to a temperature around $700^{\circ} \mathrm{C}$ when the intensity of the additionally shifted component decreases and the bulk component starts to shift back towards the initial position.

$\mathrm{Yb} 4 \mathrm{f}$ spectra acquired at a photon energy of $140 \mathrm{eV}$, after deposition, and after heating are shown in Figure 3(a). Directly after $\mathrm{Yb}$ deposition, two $\mathrm{Yb} 4 \mathrm{f}$ doublets $\left(4 \mathrm{f}_{5 / 2}\right.$, and $4 \mathrm{f}_{7 / 2}$ components, each with a branching ratio $3: 4$ and a spin-orbit splitting of $1.27 \mathrm{eV} \mathrm{[30])} \mathrm{are} \mathrm{ob-}$ served inthe $\mathrm{BE}$ range of $0-4 \mathrm{eV}$. The first doublet shows the $4 \mathrm{f}_{7 / 2}$ maximum at $\mathrm{BE}$ of $0.9 \mathrm{eV}$ and the second doublet at $1.5 \mathrm{eV}$. This spectrum looks very similar to that obtained earlier from pure $\mathrm{Yb}$ metal [31], i.e. two $\mathrm{Yb}$ 
$4 \mathrm{f}$ doublets in the $\mathrm{BE}$ range of $0-4 \mathrm{eV}$ with a separation of the $4 f_{7 / 2}$ maximum of $0.6 \mathrm{eV}$. The two $4 \mathrm{f}$ doublets observed in the pure $\mathrm{Yb}$ were defined to originate from the bulk and surface divalent $\mathrm{Yb}$ atoms. The $\mathrm{Yb} 4 \mathrm{f}$ spectrum recorded at a photon energy of $80 \mathrm{eV}$ (surface sensitive) and of $180 \mathrm{eV}$ (more bulk sensitive) are shown in Figure 3(b). These spectra clearly show variations in relative intensity between the two doublets with photon energy. The relative intensity of the first doublet with the $4 f_{7 / 2}$ at a $\mathrm{BE}$ of $0.9 \mathrm{eV}$ is found to be considerably larger at the higher photon energy while the opposite is seen to be the case for the second doublet. This indicates that the first doublet originates from bulk $\mathrm{Yb}$ atoms while the second doublet originates from the $\mathrm{Yb}$ atoms on surface which corresponds well to earlier observations on pure $\mathrm{Yb}$ metal films [31]. This finding implies that there is no reaction between $\mathrm{Yb}$ and the graphene sample after deposition at room temperature. Upon heating the $\mathrm{Yb} 4 \mathrm{f}$ spectrum is seen to exhibit distinct changes. Heating the sample at $300^{\circ} \mathrm{C}$ results both in a pronounced broadening and a shift of the $\mathrm{Yb} 4 \mathrm{f}$ spectrum to lower BE, closer to the Fermi energy, as illustrated in Figure 3(a). This broad spectrum can tentatively be de-convoluted into two overlapping doublets. The $4 f_{7 / 2}$ of the main doublet is now located at a BE of $0.5 \mathrm{eV}$ and is accompanied by a weaker doublet with the $4 \mathrm{f}_{7 / 2}$ at a $\mathrm{BE}$ of about $0.9 \mathrm{eV}$, i.e. close to the earlier bulk location. At this stage the spectrum clearly shows an absence of the surface component. Earlier studies on the formation of $\mathrm{Yb}$ silicide on $\mathrm{Si}(111)$ $[29,32]$ demonstrated that heating of samples with $\mathrm{Yb}$ deposited on $\mathrm{Si}(111)$ substrates to about $300^{\circ} \mathrm{C}-400^{\circ} \mathrm{C}$ leads to a strong reaction between $\mathrm{Yb}$ and $\mathrm{Si}$ resulting in a shift of the $\mathrm{Yb} 4 \mathrm{f}$ doublet close to the Fermi level. From this, we can therefore interpret the main component

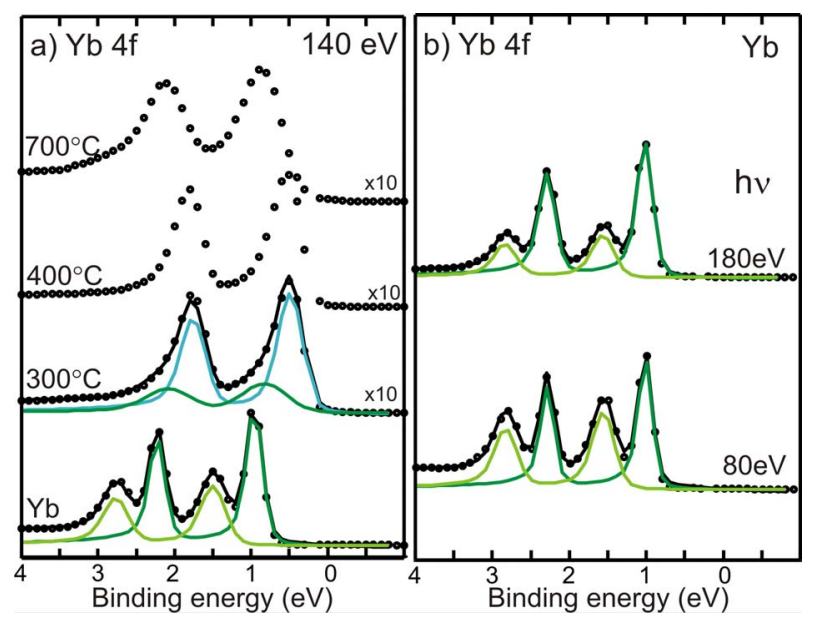

Figure 3. Yb $4 \mathrm{f}$ core level spectra collected (a) at a photon energy of $140 \mathrm{eV}$ from the graphene sample after Yb deposition and heating from $300^{\circ} \mathrm{C}$ to $700^{\circ} \mathrm{C}$; (b) at different photon energies after $\mathrm{Yb}$ deposition. observed in the $\mathrm{Yb} 4 \mathrm{f}$ spectrum recorded after heating at $300^{\circ} \mathrm{C}$ to arise from interacting $\mathrm{Yb}$ and $\mathrm{Si}$ atoms at the film-substrate interface. Moreover the relative intensity of the $\mathrm{Yb} 4 \mathrm{f}$ spectrum in the $\mathrm{BE}$ range of $0-4 \mathrm{eV}$ is found to be lower after heating suggesting that, beside the $\mathrm{Yb}$ intercalation, $\mathrm{Yb}$ may change valency [29,32], i.e. transform from divalent to trivalent $\mathrm{Yb} 4 \mathrm{f}$ that contribute to the feature at higher BE outside the probing region, or desorb from the sample during heating. No significant changes in the features of $\mathrm{Yb} 4 \mathrm{f}$ spectrum are then observed after heating up to a temperature of $700^{\circ} \mathrm{C}$ where the $\mathrm{Yb} 4 \mathrm{f}$ spectrum shows broader features and the surface component begin to reappear on the high BE tail. This suggests that the $\mathrm{Yb}-\mathrm{Si}$ bonding at the interface starts to break up at this temperature.

The above results reveal that $\mathrm{Yb}$ deposited onto graphene on $\mathrm{SiC}(0001)$ kept at room temperature do not give rise to any significant effect in $\mathrm{C} 1 \mathrm{~s}$ and $\mathrm{Si} 2 \mathrm{p}$ core level spectra. Just a slight broadening and decrease in intensity of the $\mathrm{C} 1 \mathrm{~s}$ and $\mathrm{Si} 2 \mathrm{p}$ peaks is observed, but no sign of intercalation. In stark contrast, alkali metals such as $\mathrm{Li}[6,7]$ and $\mathrm{Na}[8]$ can intercalate the graphene layer directly after deposition at room temperature as manifested by shifts of the substrate $\mathrm{C} 1 \mathrm{~s}$ and $\mathrm{Si} 2 \mathrm{p}$ core level components. In the case of $\mathrm{Yb}$, heating the sample to $300^{\circ} \mathrm{C}$ was found to be necessary to induce $\mathrm{Yb}$ intercalation under the carbon buffer layer. The $\mathrm{C} 1 \mathrm{~s}$ and $\mathrm{Si} 2 \mathrm{p}$ substrate peaks then exhibited similar shifts to lower BE, confirming the formation of a dipole layer at the substrate graphene interface, induced by interactions between $\mathrm{Yb}$ and the $\mathrm{Si}$ atoms in first $\mathrm{SiC}$ bilayer. This also corresponded well to the changes observed in the $\mathrm{Yb} 4 \mathrm{f}$ spectrum after heating to $300^{\circ} \mathrm{C}$. The $4 \mathrm{f}$ doublets then broadened and shifted closer to the Fermi level, indicating the formation of $\mathrm{Yb}-\mathrm{Si}$ bonding. The disappearance of the buffer layer (B) component in the C 1s spectrum also indicated an elimination of this layer upon $\mathrm{Yb}$ intercalation, similarly to what has been reported earlier for $\mathrm{H}$ [1-3], Li [6] and $\mathrm{Na}$ [8] intercalation of graphene on $\mathrm{SiC}(0001)$. The core-level spectra show that the $\mathrm{Yb}$ intercalated sample is fairly stable upon heating to temperatures up to $700^{\circ} \mathrm{C}$.

Valence band dispersions recorded using a photon energy of $33 \mathrm{eV}$, along the $\overline{\Gamma K}$ direction in the Brillouin zone before and after $\mathrm{Yb}$ deposition and subsequent heating are shown in Figure 4. For the clean sample, the valence band structure shows in Figure 4(a) the graphene $\sigma$ and $\pi$ bands along the $\overline{\Gamma K}$ direction. The maximum of the $\sigma$ band is located about $4.5 \mathrm{eV}$ below the Fermi level. The $\pi$ band reveals a linear dispersion close to the $\bar{K}$ point and a band minimum located around $9.0 \mathrm{eV}$ below the Fermi level at the $\bar{\Gamma}$ point. After $\mathrm{Yb}$ deposition, there are no significant changes in the dispersion of the graphene $\sigma$ and $\pi$ bands, as seen in Figure 4(b). Four ad- 
ditional levels originating from $\mathrm{Yb} 4 \mathrm{f}$ states are hoever observed between one and three eV below the Fermi level, see a close-up image at the lowermost right corner. These are the localized states representing the $4 \mathrm{f}_{5 / 2}$ and $4 \mathrm{f}_{7 / 2}$ levels of the bulk and surface $\mathrm{Yb}$ atoms, similar to what is observed in the $\mathrm{Yb} 4 \mathrm{f}$ spectrum in Figure 3. After heating the sample at $400^{\circ} \mathrm{C}$, an additional $\pi$ band appears, most clearly in the region of $\mathrm{k}_{/ /}$from 0.4 to $1.6 \AA^{-1}$, see the inset at the right corner of Figure (4), while at $\bar{\Gamma}$ the overlap is so strong so they look more like one broad band.
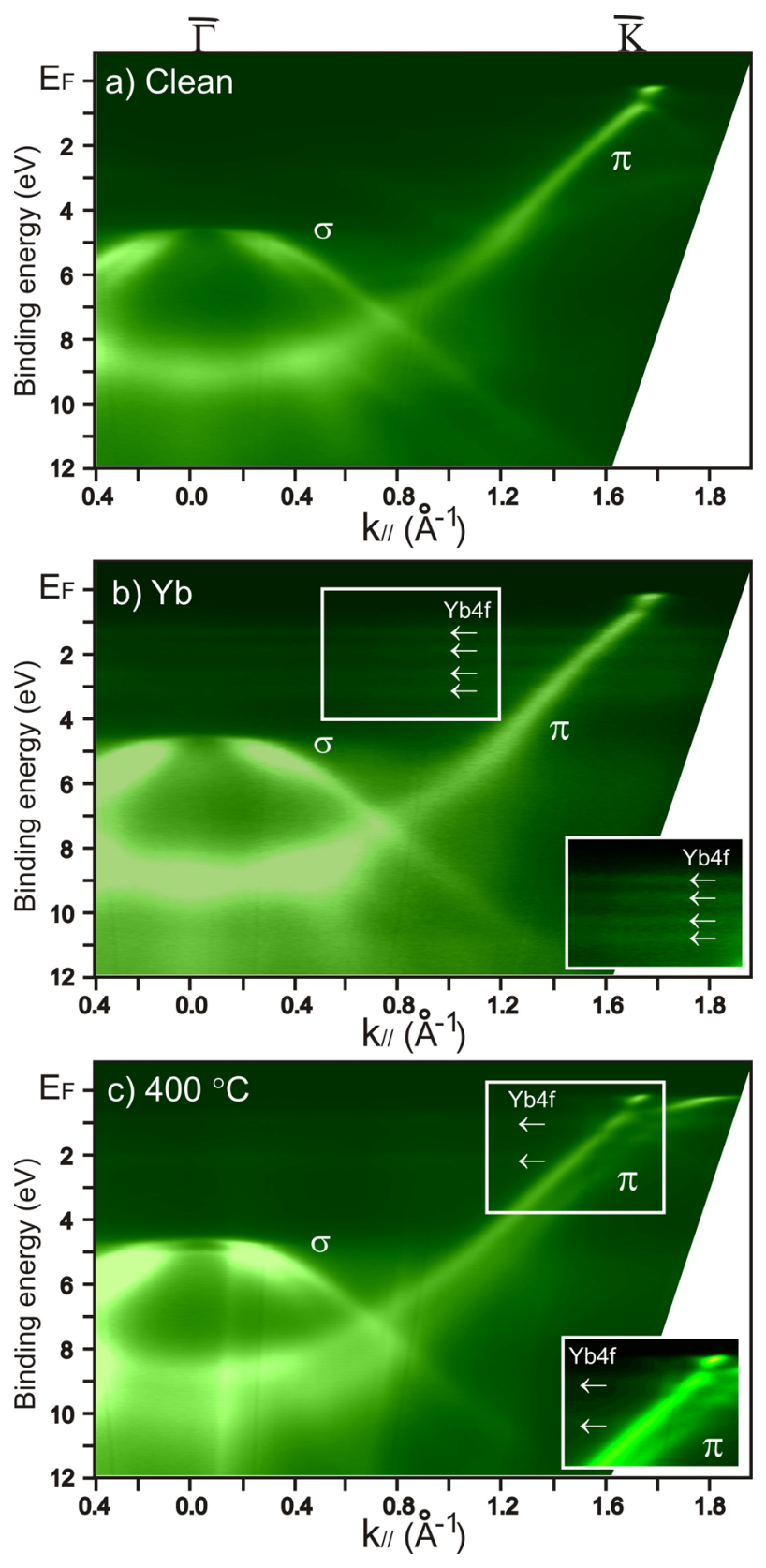

Figure 4. Electronic band structure along $\overline{\Gamma K}$ direction for the graphene sample (a) before and (b) after $\mathrm{Yb}$ deposition and (c) heating at $400^{\circ} \mathrm{C}$.
No shift or change in the $\sigma$ band can be observed after heating. The number of $\pi$ bands observed from graphene grown on a $\mathrm{SiC}(0001)$ substrate can be used to determine the number of graphene layers [27]. Hence, the increase in the number of $\pi$ bands observed in this case may simply imply an increase in the number of graphene layers from one to two layers due to $\mathrm{Yb}$ intercalation, in agreement with the results from angle-integrated photoemission discussed above. The $\pi$ bands collected around the $\bar{K}$ point at higher energy and angular resolution are shown in Figure 5. The as-grown sample exhibits a single $\pi$ band with the Dirac point located about $0.4 \mathrm{eV}$ below the Fermi level, which are characteristics of monolayer graphene on SiC(0001), see Figure 5(a). The shift of the Dirac point away from the Fermi level has been interpreted to be due to interactions with the substrate $[4,33]$. Deposition of $\mathrm{Yb}$ affects the graphene $\pi$ bands very little, as seen in Figure 5(b). Only the intensity of the bands was slightly lower than for the clean sample. No shift of the Dirac point could thus be detected after deposition of $\mathrm{Yb}$ on graphene kept at room temperature. This is contrary to results for alkali metals (Li [5], $\mathrm{Na}$ [7], K [34], Rb and Cs [15]) deposited on graphene at room temperature where fairly large shifts of the Dirac point to further away from the Fermi level were observed directly after deposition, due to electron doping of the graphene. However, after $\mathrm{Yb}$ deposition and heating at $400^{\circ} \mathrm{C}$ and $500^{\circ} \mathrm{C}$, respectively, two $\pi$ bands appear as shown in Figures 5(c) and (d). This indicates the formation of two graphene layers that we interpret to be due to $\mathrm{Yb}$ intercalation. The two $\pi$ bands are quite distinct and exhibit different Dirac points located at around $0.4 \mathrm{eV}$ and $1.9 \mathrm{eV}$ below the Fermi level, respectively. This downward shift by about $1.5 \mathrm{eV}$ of the $\pi$ band is interpreted to originate from electron charge transfer from $\mathrm{Yb}$ atoms intercalated at the interface to unoccupied graphene $\pi$ states. It is only after heating at temperatures when $\mathrm{Yb}$ $\mathrm{Si}$ bonding can detected in the core level spectra that this intercalation and doping of graphene layers can be observed. It deserves to be noted that the dispersion and location of the $\pi$ band that we obtain close to the $\bar{K}$ point

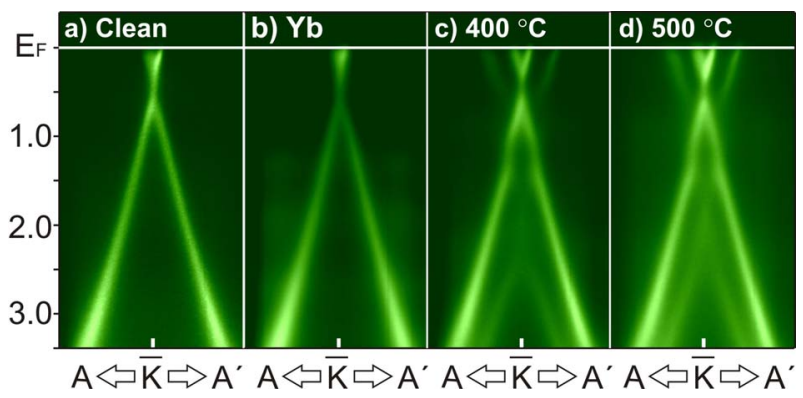

Figure 5. $\pi$-band dispersion around the $\bar{K}$ point recorded from the graphene sample (a) before and (b) after $\mathrm{Yb}$ depostion, after heating (c) at $400^{\circ} \mathrm{C}$ and d) $500^{\circ} \mathrm{C}$. 
after $\mathrm{Yb}$ intercalation of graphene on $\mathrm{SiC}(0001)$ looks quite different compared to earlier mappings of the $\pi$ band after $\mathrm{Yb}$ intercalation in graphite [20] and in monolaye graphene on $\mathrm{Ni}(111)$ [21]. In both cases the $\pi$ band was located about four to five $\mathrm{eV}$ below the Fermi level and did not show a linear dispersion and for the $\mathrm{Gr} / \mathrm{Yb} / \mathrm{Ni}(111)$ system it moreover showed a splitting into two branches around the $\bar{K}$ point.

By using LEEM and $\mu$-LEED it is possible to probe in a real-time the changes induced during $\mathrm{Yb}$ deposition and heating. A LEEM image of the clean as-grown graphene sample at a field of view (FOV) of $10 \mu \mathrm{m}$ is shown in Figure 6(a). This image reveals the small graphene flakes/domains normally obtained when preparing graphene on $\mathrm{SiC}(0001)$ by heating in situ. A dominant coverage of $1 \mathrm{ML}$ graphene domains had been determined from the $\mathrm{G} / \mathrm{SiC}$ and $\mathrm{B} / \mathrm{SiC}$ peak intensity ratios obtained in recorded $\mathrm{C}$ 1s spectra, and was confirmed by the single minimum observed in the electron reflectivity curve extracted versus electron energy. Deposition of $\mathrm{Yb}$ on the sample at room temperature did not induce any

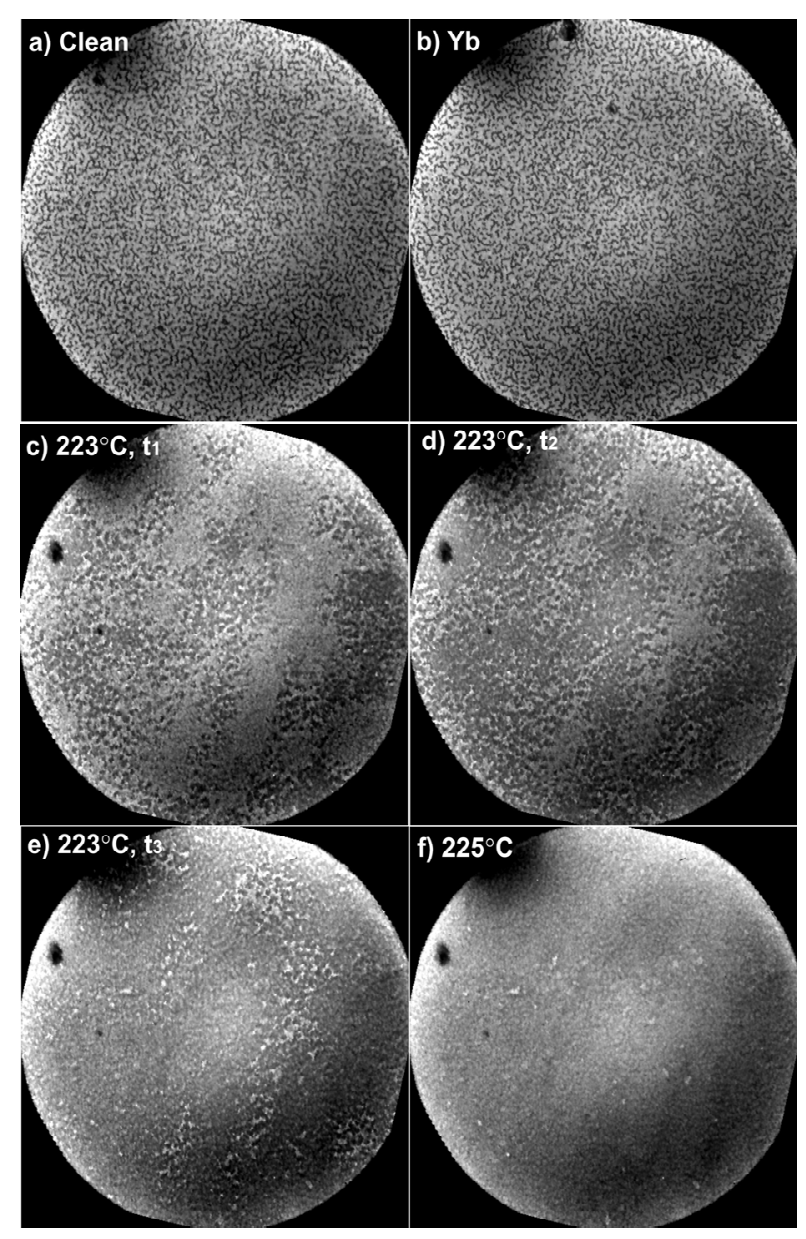

Figure 6. LEEM image with FOV of $10 \mu \mathrm{m}$ recorded from the graphene sample (a) before and (b) after $\mathrm{Yb}$ deposition, and after heating (c)-(e) at $223^{\circ} \mathrm{C}$ and (f) $225^{\circ} \mathrm{C}$. significant changes in the LEEM image, as illustrated in Figure 6(b). Only the small graphene flakes are still visible and there are no metal droplets formed on the sample. During the subsequent heating LEEM images were recorded in the real-time. No pronounced changes were observed in the images until a temperature around $220^{\circ} \mathrm{C}$ was reached, see Figures 6(c)-(e). These three images were collected at half a second intervals at a temperature of $223^{\circ} \mathrm{C}$ and show that, at the same electron kinetic energy of $2 \mathrm{eV}$, two types of domains with different contrasts, bright and dark gray, are now discernable. It is also clearly seen that the relative amount of these two domain types change with time at this temperature. The darker and smoother gray domains appear to grow and cover a larger part of the surface. When reaching a temperature of $225^{\circ} \mathrm{C}$, the surface looks more or less homogeneous as seen in Figure 6(f). No more pronounced morphology changes were observed in the LEEM images when increasing the temperature further up to $700^{\circ} \mathrm{C}$. Electron reflectivity curves were extracted versus electron energy, $I(V)$ curves, from the LEEM images collected. Before $\mathrm{Yb}$ deposition the $I(V)$ curve exhibited a single minimum/dip which represents monolayer graphene. After $\mathrm{Yb}$ deposition, the $I(V)$ curve was fairly flat and exhibited two weak broad minima. After heating the sample at $260^{\circ} \mathrm{C}$, the $I(V)$ curve was still very flat but exhibited an additional weak minimum. We have at present no clear interpretation of these $I(V)$ curves, but it is likely that the interpretation of the $I(V)$ curves within the rather simplistic kinematic diffraction model that is typically assumed, also known as quantum confinement contrast [35], has to be replaced by a more realistic description taking into account the particular electronic structure of the substrate and interface region as modified by the intercalant. This can, in principle, be achieved within the framework of ab-initio scattering theory, which properly accounts for band structure effects in the electron reflectivity [36], but which is certainly beyond the scope of the present paper.

The $\mu$-LEED pattern collected from the clean sample before $\mathrm{Yb}$ deposition in Figure 7(a) shows sharp $(1 \times 1)$ graphene spots surrounded by $(6 \sqrt{3} \times 6 \sqrt{3}) R 30^{\circ}$ buffer layer spots. The $(1 \times 1) \mathrm{SiC}$ substrate spots are also detected but their intensities are quite low at this selected

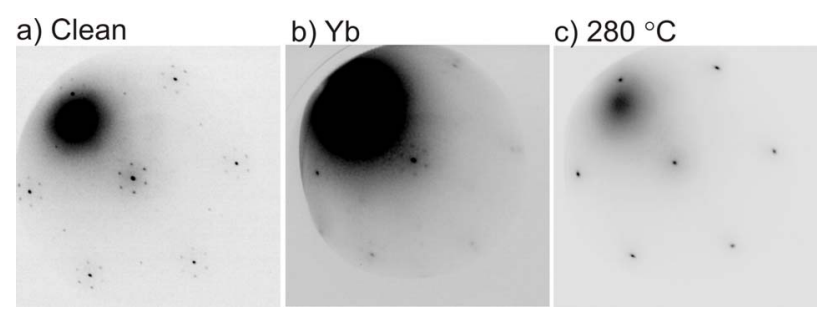

Figure 7. $\mu$-LEED patterns collected at electron energy of $50 \mathrm{eV}$ for (a) before and (b) after $\mathrm{Yb}$ deposition, and after heating (c) at $280^{\circ} \mathrm{C}$. 
energy. The rotation of the graphene spots with respect to the substrate spots indicates that the graphene layer is rotated an angle of $30^{\circ}$ with the $\mathrm{SiC}$ substrate. The darkshadow appearing in the uppermost left corner in the $\mu$-LEED images arises from secondary electrons and is unavoidable since the instrument used has no energy filter. After $\mathrm{Yb}$ deposition at room temperature, the $\mu$ LEED pattern became fainter and the graphene spots appear more diffuse, as illustrated in Figure 7(b). The buffer layer and $\mathrm{SiC}$ substrate spots are no longer clearly visible. The $\mu$-LEED pattern recorded again after heating at $280^{\circ} \mathrm{C}$, see Figure $7(\mathbf{c})$, shows sharp graphene spots but now the buffer layer and substrate spots have completely disappeared. This disappearance of the buffer layer $\mu$-LEED spots is in accordance with the absence of the buffer layer (B) component in the $\mathrm{C}$ 1s spectra in Figure 1(a) and supports our interpretation of elimination/transformation of the buffer layer by $\mathrm{Yb}$ intercaltion.

\section{Conclusion}

We have shown that the rare earth metal, $\mathrm{Yb}$, can intercalate into graphene grown on $\mathrm{SiC}(0001)$. PES and AR$\mathrm{PES}$ measurements reveal that, after $\mathrm{Yb}$ deposition with the sample at room temperature, $\mathrm{Yb}$ stays on top of the graphene and does not intercalate or distort the graphene layer. This is clearly revealed by the preserved features in the $\mathrm{C} 1 \mathrm{~s}$ and Si 2p core level spectra, the band structure, and by the $\mathrm{Yb} 4 \mathrm{f}$ spectrum, which shows both a bulk and a surface shifted $4 \mathrm{f}$ doublet. These results are different to what has been observed earlier for the alkali metals $\mathrm{Li}$ and $\mathrm{Na}$ that when deposited on graphene at room temperature induced intercalation. After subsequent heating, $\mathrm{Yb}$ intercalation was found to occur as manifested by the appearance of clear shifts of the substrate $\mathrm{C} 1 \mathrm{~s}$ and $\mathrm{Si} 2 \mathrm{p}$ components and pronounced changes in energy and shape of the $\mathrm{Yb} 4 \mathrm{f}$ doublets. Real-time LEEM measurements indicated intercalation to occur at a sample temperature around $220^{\circ} \mathrm{C}$. The results show that $\mathrm{Yb}$ penetrates through the graphene and the buffer layer and forms bonds to $\mathrm{Si}$ at the substrate interface. The $\mathrm{Yb}$ intercalation results in an elimination of the buffer layer as indicated by the absence of a buffer layer component in the $\mathrm{C} 1 \mathrm{~s}$ spectrum and buffer layer spots in the $\mu$ LEED pattern. The intercalant $\mathrm{Yb}$ decouples the buffer layer from the substrate and transforms it into a second graphene layer contributing to a second $\pi$ band as observed in the ARPES spectra. However, these two graphene layers contain different amounts of doping as seen from the different energy positions of the Dirac points of these two $\pi$ bands. The $\mathrm{Yb}$ intercalated graphene sample is found to be stable up to a heating temperature around $700^{\circ} \mathrm{C}$, when $\mathrm{Yb}$ starts to desorb from the sample, and the core level spectra are observed to begin to transform back to their initial shapes.

\section{Acknowledgements}

The authors gratefully acknowledge support from the European Science Foundation.

\section{REFERENCES}

[1] C. Virojanadara, A. A. Zakharov, R. Yakimova and L. I. Johansson, "Buffer Layer Free Large Area Bi-Layer Graphene on SiC(0001)," Surface Science, Vol. 604, 2010, pp. L4-L7.

[2] C. Riedl, C. Coletti, T. Iwasaki, A. A. Zakharov and U. Starke, "Quasi-Free-Standing Epitaxial Graphene on $\mathrm{SiC}$ Obtained by Hydrogen Intercalation," Physical Review Letters, Vol. 103, No. 24, 2009, Article ID: 246804. doi:10.1016/i.susc.2009.11.011

[3] S. Watcharinyanon, C. Virojanadara, J. R. Osiecki, A. A. Zakharov, R. Yakimova, R. I. G. Uhrberg and L. I. Johansson, "Hydrogen Intercalation of Graphene Grown on 6HSiC(0001)," Surface Science, Vol. 605, No. 17-18, 2011, pp. 1662-1668. doi:10.1016/j.susc.2010.12.018

[4] K. V. Emtsev, F. Speck, T. Seyller, L. Ley and J. D. Riley, "Interaction, Growth, and Ordering of Epitaxial Graphene on SiC(0001) Surfaces: A Comparative Photoelectron Spectroscopy Study," Physical Review B, Vol. 77, No. 15, 2008, Article ID: 155303. doi:10.1103/PhysRevB.77.155303

[5] F. Speck, J. Jobst, F. Fromm, M. Ostler, D. Waldmann, M. Hundhausen, H. B. Weber and T. Seyller, "The QuasiFree-Standing Nature of Graphene on H-Saturated SiC (0001)," Applied Physics Letters, Vol. 99, No. 12, 2011, Article ID: 122106. doi:10.1063/1.3643034

[6] C. Virojanadara, S. Watcharinyanon, A. A. Zakharov and L. I. Johansson, "Epitaxial Graphene on $6 \mathrm{H}-\mathrm{SiC}$ and $\mathrm{Li}$ Intercaltion," Physical Review B, Vol. 82, No. 20, 2010, Article ID: 205402. doi:10.1103/PhysRevB.85.205402

[7] C. Virojanadara, A. A. Zakharov, S. Watcharinyanon, R. Yakimova and L. I. Johansson, "A LEEM and XPEEM Study of Li Intercalated into Graphene on $\mathrm{SiC}(0001)$," New Journal of Physics, Vol. 12, 2010, Article ID: 125015.

[8] S. Watcharinyanon, L. I. Johansson, C. Xia and C. Virojanadara, "Changes in Structural and Electronic Properties of Graphene Grown on $6 \mathrm{H}-\mathrm{SiC}(0001)$ Induced by $\mathrm{Na}$ Deposition," Journal of Applied Physics, Vol. 111, No. 8, 2012, Article ID: 083711. doi:10.1063/1.4704396

[9] A. Sandin, T. Jayasekera, J. E. Rowe, K. W. Kim, M. B. Nardelli and D. B. Dougherty, "Multiple Coexisting Intercalation Structures of Sodium in Epitaxial Graphene-SiC Interfaces," Physical Review B, Vol. 85, No. 12, 2012, Article ID: 125410 . doi:10.1103/PhysRevB.85.125410

[10] I. Gierz, T. Suzuki, R. T. Weitz, D. S. Lee, B. Krauss, C. Riedl, U. Starke, H. Höchst, J. H. Smet, C. R. Ast and K. Kern, "Electronic Decoupling of an Epitaxial Ggraphene Monolayer by Gold Intercalation," Physical Review B, Vol. 81, No. 23, 2010, Article ID: 235408. doi:10.1103/PhysRevB.81.235408

[11] C. Xia, S. Watcharinyanon, A. A. Zakharov, R. Yakimova, L. Hultman, L. I. Johansson and C. Virojanadara, "Detailed Studies of Si Intercalation/De-intercalation of Graphene on 6H-SiC(0001)," Physical Review B, Vol. 85, No. 
4, 2012, Article ID: 045418. doi:10.1103/PhysRevB.85.045418

[12] K. V. Emtsev, A. A. Zakharov, C. Coletti, S. Forti and U. Starke, "Ambipolar Doping in Quasifree Epitaxial Graphene on $\mathrm{SiC}(0001)$ Controlled by Ge Intercalation," Physical Review B, Vol. 84, No. 12, 2011, Article ID: 125423. doi:10.1103/PhysRevB.84.125423

[13] A. L. Walter, K.-J. Jeon, A. Bostwick, F. Speck, M. Ostler, T. Seyller, L. Moreschini, Y. S. Kim, Y. J. Chang, K. Horn and E. Rotenberg, "Highly p-Doped Epitaxial Graphene Obtained by Fluorine Intercalation," Applied Physics Letters, Vol. 98, No. 18, 2011, Article ID: 184102. doi:10.1063/1.3586256

[14] L. Meng, R. Wu, H. Zhou, G. Li, Y. Zhang, L. Li, Y. Wang and H.-J. Gao, "Silicon Intercalation at the Interface of Graphene and $\operatorname{Ir}(111)$, , Applied Physics Letters, Vol. 100, No. 8, 2012, Article ID: 083101. doi:10.1063/1.3687688

[15] J. Sanchez-Barriga, A. Varykhalov, M. R. Scholz, O. Rader, D. Marchenko, A. Rybkin, A. M. Shikin and E. Vescovo, "Chemical Vapour Deposition of Graphene on Ni (111) and Co(0001) and Intercalation with Au to Study Dirac-Cone Formation and Rashba Splitting," Diamond and Related Materials, Vol. 19, 2010, pp. 734-741.

[16] S. Watcharinyanon, C. Virojanadara and L. I. Johansson, "Rb and Cs Deposition on Epitaxial Graphene Grown on 6H-SiC(0001)," Surface Science, Vol. 605, No. 21-22, 2011, pp. 1918-1922. doi:10.1016/j.susc.2011.07.007

[17] A. L. Walter, A. Bostwick, K.-J. Jeon, F. Speck, M. Ostler, T. Seyller, L. Moreschini, Y. J. Chang, M. Polini, R. Asgari, A. H. MacDonald, K. Horn and E. Rotenberg, "Effective Screening and the Plasmaron Bands in Graphene," Physical Review B, Vol. 84, No. 8, 2011, Article ID: 085410. doi:10.1103/PhysRevB.84.085410

[18] Private Communication with E. Rotenberg, 2011.

[19] T. Loftus, J. R. Bochinski and T. W. Mossberg, "Magnetic Trapping of Ytterbium and the Alkaline-Earth Metals," Physical Review A, Vol. 66, No. 1, 2002, Article ID: 013411. doi:10.1103/PhysRevA.66.013411

[20] S. L. Molodtsov, C. Laubschat, M. Richter, T. Gantz and A. M. Shikin, "Electronic Struture of Eu and Yb Graphite Intercaltion Compounds," Physical Review B, Vol. 53, No. 24, 1996, pp. 16621-16629. doi:10.1103/PhysRevB.53.16621

[21] A. M. Shikin, M. V. Poigin, Y. S. Dedkov, S. L. Molodtsov and V. K. Adamchuk, "Formation of Intercalate-Like System of Graphite-Yttibium Monolayers on the Ni(111) Surface," Physics of the Solid State, Vol. 42, No. 6, 2000, pp. 1170-1175. doi:10.1134/1.1131335

[22] T. E. Weller, M. Ellerby, S. Saxena, R. P. Smith and N. T. Skipper, "Superconductivity in the Intercalated Graphite Compounds C6Yb and C6Ca," Nature Physics, Vol. 1, No. 1, 2005, pp. 39-41. doi:10.1038/nphys0010

[23] C. Virojanadara, M. Syväjärvi, R. Yakimova, L. I. Johansson, A. A. Zakharov and T. Balasubramanian, "Homogeneous Large-Area Graphene Layer Growth on $6 \mathrm{H}-$ SiC(0001)," Physical Review B, Vol. 78, No. 24, 2008, Article ID: 245403. doi:10.1103/PhysRevB.78.245403

[24] T. Ohta, F. E. Gabaly, A. Bostwick, J. L. McChesney, K.
V. Emtsev, A. K. Schmid, T. Seyller, K. Horn and E. Rotenberg, "Morphology of Graphene Thin Film Growth on $\mathrm{SiC}(0001)$," New Journal of Physics, Vol. 10, No. 2, 2008, Article ID: 023034. doi:10.1088/1367-2630/10/2/023034

[25] L. N. Srivastava, G. He, R. M. Feenstra and P. J. Fisher, "Comparing of Graphene Formation on C-Face and SiFace SiC(0001) Surfaces," Physical Review B, Vol. 82, No. 23, 2010, Article ID: 235406. doi:10.1103/PhysRevB.82.235406

[26] C. Virojanadara, R. Yakimova, A. A. Zakharov and L. I. Johansson, "Large Homogeneous Mono/Bi-layer Graphene on $6 \mathrm{H}-\mathrm{SiC}(0001)$ and Buffer Layer Elimination," Journal of Physics D, Vol. 43, No. 37, 2010, Article ID: 374010. doi:10.1088/0022-3727/43/37/374010

[27] T. Ohta, A. Bostwick, J. L. McChesney, T. Seyller, K. Horn and E. Rotenberg, "Interlayer Interaction and Electronic Screening in Multilayer Graphene Investigated with Angle-Resolved Photoemission Spectroscopy," Physical Review Letters, Vol. 98, No. 20, 2007, Article ID: 206802. doi:10.1103/PhysRevLett.98.206802

[28] P. H. Mahowald, D. J. Friedman, G. P. Carey, K. A. Bertness and J. J. Yeah, "Computer Program for Photoemission Data Analysis and Display," Journal of Vacuum Science \& Technology A, Vol. 5, No. 5, 1987, pp. 29822985. doi:10.1116/1.574244

[29] R. Hofmann, W. A. Henle and F. P. Netzer, "Electronic Structure of Epitaxial Yb Silicide," Physical Review B, Vol. 46, No. 7, 1992, pp. 3857-3864. doi:10.1103/PhysRevB.46.3857

[30] E.-J. Cho, J.-S. Chung, S.-J. Oh, S. Suga, M. Taniguchi, A. Kakizaki, A. Fujimori, H. Kato, T. Miyahara, T. Suzuki and T. Kasuya, "Surface Core-Level Shifts and Electronic Structures of $\mathrm{Yb}$ Compounds Studied with Use of Photoemission Spectrocopy," Physical Review B, Vol. 47, No. 7, 1993, pp. 3933-3943. doi:10.1103/PhysRevB.47.3933

[31] F. Gerken, A. S. Flodstrom, J. Barth, L. I. Johansson and C. Kunz, "Surface Core Level Shifts of the Lanthanide Metal Ce58-Lu71: A Comprehensive Experiment Study," Physica Scripta, Vol. 32, No. 1, 1985, pp. 43-57. doi:10.1088/0031-8949/32/1/006

[32] C. Wigren, J. N. Andersen, R. Nyholm and U. O. Karlsson, "Growth and Epitaxy of Yb Silicides on $\mathrm{Si}(111)$," Journal of Vacuum Science \& Technology A, Vol. 9, 1991, pp. 1942-1945. doi:10.1116/1.577549

[33] J. Ristein, S. Mammadov and T. Seyller, "Origin of Doping in Quasi-Free-Standing Graphene on SiC," Physical Review Letters, Vol. 108, No. 24, 2012, Article ID: 246104. doi:10.1103/PhysRevLett.108.246104

[34] A. Bostwick, T. Ohta, T. Seyller, K. Horn and E. Rotenberg, "Quasiparticle Dynamics in Graphene," Nature Physics, Vol. 3, No. 1, 2007, pp. 36-40. doi:10.1038/nphys477

[35] M. S. Altman, W. F. Chung and C. H. Liu, "LEEM Phase Contrast," Surface Review and Letters, Vol. 5, No. 6, 1998, pp. 1129-1141. doi:10.1142/S0218625X98001468

[36] J. I. Flege, A. Meyer, J. Falta and E. E. Krasovskii, "Self-Limited Oxide Formation in Ni(111) Oxidation," Physical Review B, Vol. 84, No. 11, 2012, Article ID: 115441 . doi:10.1103/PhysRevB.84.115441 